\title{
Medical, Political, and Economic Considerations for the Use of MAC for Endoscopic Sedation: Big Price, Little Justification?
}

\author{
Basavana Goudra $^{1}$ (D) Preet Mohinder Singh ${ }^{2}$ (C) Gary R. Lichtenstein ${ }^{3}$
}

Published online: 16 July 2020

(c) Springer Science+Business Media, LLC, part of Springer Nature 2020

\begin{abstract}
The last few decades of gastrointestinal (GI) endoscopy have seen phenomenal growth. In many aspects, GI endoscopy has led the field of nonsurgical interventional medicine. In many aspects, this growth is facilitated by advancements in sedationboth drugs and techniques. Unfortunately, the topic of GI endoscopy sedation is also mired in many controversies, mainly emanating from the cost of anesthesia providers. While no one debates their role in the majority of advanced endoscopic procedures, the practice of universal propofol sedation in the USA, delivered by anesthesia providers, needs a closer look. In this review, medical, political, and economic considerations of this important topic are discussed in a very frank and honest way. While such ubiquitous propofol use has increased satisfaction of both patients and gastroenterologists, there is little justification. More importantly, going by the evidence, there is even less justification for the mandated anesthesia providers use for such delivery. Unfortunately, the FDA could not be convinced otherwise. The new drug fospropofol met the same fate. Approval of SEDASYS ${ }^{\circledR}$, the first computer-assisted personalized sedation system, was a step in the right direction, nevertheless an insufficient step that failed to takeoff. As a result, in spite of years of research and efforts of many august societies, the logjam of balancing cost and justification of propofol sedation has continued. We hope that recent approval of remimazolam, a novel benzodiazepine, and potential approval of oliceridine, a novel short-acting opioid, might be able to contain the cost without compromising the quality of sedation.
\end{abstract}

Keywords GI endoscopy $\cdot$ Sedation $\cdot$ Propofol $\cdot$ Sedasys $\cdot$ Oliceridine $\cdot$ Remimazolam

There are very few areas in medicine that are as challenged, examined, and debated as is endoscopic sedation; the reasons are many with the most prominent being the cost of

Basavana Goudra

goudrab@uphs.upenn.edu

Gary R. Lichtenstein

Gary.Lichtenstein@pennmedicine.upenn.edu

1 Perelman School of Medicine, Hospital of the University of Pennsylvania, 680 Dulles, 3400 Spruce Street, Philadelphia, PA 19104, USA

2 Department of Anesthesiology, Washington University in Saint Louis, 660 South Euclid Avenue, St Louis, MO 63110, USA

3 Division of Gastroenterology, Department of Medicine, Center for Inflammatory Bowel Disease, Raymond and Ruth Perelman School of Medicine of the University of Pennsylvania, GI Administration Offices, 7th Floor South Pavilion, Room 753, Perelman Center for Advanced Medicine, 3400 Civic Center Boulevard, Philadelphia, PA 19104-4283, USA anesthesia providers. To be more precise, it is the question of cost versus benefit. Arguably, monitored anesthesia care (MAC) has become synonymous with propofol sedation. We propose three questions: Is propofol the sine qua non for endoscopic sedation? Can non-anesthesia providers provide safe sedation with propofol? Finally, do all patients require or benefit from deep sedation? In the ensuing paragraphs, we will make an unbiased assessment of the available evidence.

\section{Brief History of Unsedated and Sedated Endoscopy}

Although as of today, sedated endoscopy is nearly universal in the USA [1], a few decades ago and even now, unsedated endoscopy is common in many countries around the world. In a questionnaire-based study performed in Europe, Ladas et al. found that $<25 \%$ of patients are sedated for routine diagnostic upper GI endoscopy [2]. The majority were not even given a choice, and no written consent was obtained. 
Propofol was used in $<50 \%$ of sedated patients. Diagnostic upper GI endoscopy under topical pharyngeal anesthesia using 5\% lidocaine was associated with significant changes in pulse rate, respiratory rate, and blood pressure (suggesting that discomfort was present), but not mean oxygen saturation $\left(\mathrm{SpO}_{2}\right)$. Furthermore, the expected level of discomfort during endoscopy was not statistically different between pre- and post-procedure questioning, with three-fourths of patients expressing their willingness to undergo repeat nonsedated endoscopy in the future [3]. The use of ultrathin endoscopes has facilitated the use of unsedated endoscopy for procedures such as screening and surveillance of gastric cancer [4]. In 140 patients undergoing upper GI endoscopy with ultrathin devices, only $16(11.4 \%)$ required sedation.

Colonoscopy started as an unsedated procedure; both polyp detection rates and patient comfort were acceptable when colonoscopy was performed with no sedation, even in those who were considered high risk for anesthesia [5]. In contemporary practice, the use of unsedated colonoscopy is primarily patient driven [6].

Diazepam was one of the earliest agents used for endoscopic sedation. Although it increased patient satisfaction compared with placebo and is believed to be safe from a cardiovascular and respiratory standpoint, patient acceptability for the procedure was significantly higher when using the alternative midazolam [7, 8]. Thus, the era of shorter-acting drugs with superior amnesia and rapid recovery started. To further improve patient satisfaction, shorter-acting opioids such as fentanyl were introduced. Paradoxical excitation was an occasional issue with midazolam. Although respiratory depression was a major adverse effect, the availability of a specific antagonist to midazolam (flumazenil) provided confidence to the health care providers who were administering these drugs to produce "moderate sedation" or "traditional sedation" [9].

\section{Explosive Growth of GI Endoscopic Volume and Complexity}

Alongside new drug inventions and improved sedation techniques, enhancements in endoscopist skills and devices enabled the performance of significantly more complicated procedures. Originally, most advanced procedures were performed under conscious sedation with deep sedation/general anesthesia used infrequently [10]. In a retrospective analysis authored by Etzkorn et al., in 1998, of 1200 ERCP procedures performed over a 2-year period, only 65 patients required general anesthesia, with substance abuse as the major indication. They suggested that general anesthesia may be considered on a limited basis in order to ensure a successful and safe ERCP if the patients are likely to fail conscious sedation. Clearly, the list of advanced GI endoscopic procedures has grown exponentially in the last 2 decades. An analysis by iData Research indicates that about 75 million gastrointestinal endoscopies are performed each year in the USA alone, of which $>19$ million are colonoscopies [11].

\section{Era of MAC}

The advent of propofol sedation heralded a massive change in sedation approach. The use of the term "MAC" facilitates billing, indicating that the anesthesia provider is present to continually monitor the patient. It replaced the earlier term "standby" that was confusing to the insurance companies making payment. The term does not describe the type of drug administered used for sedation. In fact, there may not be any sedative administered [12].

Propofol was soon found to be an ideal sedative for a short-intermediate procedure such as GI endoscopy that generally causes no significant post-procedural pain or discomfort. In countries such as the USA, where patient satisfaction and high throughput are paramount, or more importantly any dissatisfaction from the patients is not taken kindly by the employers, propofol was beneficial. Caregivers could assure patients that they would not feel any pain or discomfort, would experience very little nausea, and would awake rapidly after the procedure, all qualities that facilitate early discharge and a rapid postprocedure turnover.

\section{Challenges to MAC}

\section{Medical}

Some of the benefits of MAC are undisputed. These patients typically recover more rapidly and are ready for discharge sooner than those sedated with midazolam-fentanyl combination [13-16]. This particular benefit of propofol sedation is likely to offset some of the costs resulting from decreased throughput and longer waitlists that are likely with conscious sedation [17]. The differences in the pharmacokinetics of propofol in comparison with midazolam-fentanyl include a faster onset of action, occurring in seconds rather than minutes [18, 19]. As a result, the time from injection of sedative and intubation is likely to be shorter with significant cost and time savings.

Patient satisfaction with propofol sedation is described as both similar and superior to conscious sedation. Tabir et al. did not find any major association between the sedation method and the level of satisfaction with flexible 
upper gastrointestinal endoscopy. Hey et al. compared 137 patients who were sedated with pethidine/midazolam with 104 patients receiving propofol. As expected, patients receiving propofol recovered faster with a significantly shorter procedure duration [20]. Koshy et al. demonstrated a statistically significant improvement in comfort and sedation score with propofol compared to midazolam and meperidine in GI endoscopy [21]. Similarly, in a prospective, randomized controlled trial Vargo et al. found higher satisfaction in the propofol group

Nevertheless, the safety of MAC needs careful consideration. Our own retrospective analysis of 5 years of data (73,029 GI endoscopic procedures) clearly suggested a strong association between the type of sedation as well as numerous patient factors and the frequency of adverse events. In addition to physical status classification assessed by anesthesiologists and the procedure type, propofol sedation was associated with a higher incidence of adverse events including cardiac arrest [22, 23]. These events were significantly less frequent in patients who were administered conscious sedation, typically involving midazolam and fentanyl. Another metanalysis authored by Wadhwa et al. [24] included 2518 patients, of whom 1324 received propofol, whereas 1194 received midazolam, meperidine, pethidine, remifentanil, and/or fentanyl. In comparison with conscious sedation, the pooled odds ratio with the use of propofol for developing hypoxia for all the procedures combined was 0.82 and for developing hypotension was 0.92 . Similarly, in a retrospective, nonrandomized, observational cohort study, Vargo et al. found a higher incidence of serious adverse events in patients sedated by anesthesia professionals, who typically used propofol, than endoscopist-directed midazolam/fentanylbased sedation [25].

\section{Political}

In spite of significant evidence for the safety of propofol when administered by non-anesthesia providers, it continues to be used officially only by anesthesia groups for GI endoscopic sedation, at least in the USA. This is related to the FDA label for propofol that dictates its use only by persons trained in the administration of general anesthesia. Some enthusiastic gastroenterologists have questioned this instruction repeatedly, and some societies have issued statements in this regard. In 2005, Rex et al. published a study in the journal Gastroenterology (the premier GI journal published by the American Gastroenterological Association [AGA]) involving 36,743 cases of nurse-administered propofol sedation performed at 3 centers. They attested to the safety of propofol sedation by non-anesthesia providers with very few respiratory events in any of the centers [26].
In a study involving propofol administration by general practitioner sedationists, Clarke et al. [27], reported a very low incidence of respiratory arrest, airway obstruction, hypoxia requiring intervention, hypotension, and death. Encouraged by similar results [28], the AGA concluded in an AGA Institute Review of Endoscopic Sedation in 2007 "that, if used carefully, gastroenterologist directed propofol is medicolegally reasonable, with appropriate endoscopist training, patient selection, and adherence to protocols for administration, as well as compliance with institutional and local regulations." [29] Later, in 2008, the Canadian Association of Gastroenterology endorsed the formal training guidelines required for propofol administration published by the American Society for Gastrointestinal Endoscopy, concluding that appropriately trained endoscopic nurses can also administer propofol for endoscopy under diligent monitoring. It cautioned against inadvertent bolus doses with attendant deep anesthesia [17].

Nevertheless, in the USA, non-anesthesia provider administered propofol never was widely popular except in research settings with a small group of enthusiastic gastroenterologists. As noted in the 2017 AGA institute review [30,] many obstacles were medicolegal rather than medical. Balancing the patient's expectations for a painless endoscopy and the risks of potential deep sedation while trying to accomplish this objective was a constant worry. Having seen the benefits of propofol sedation with consequent increasing patient compliance for screening procedures, endoscopists were unwilling to abandon this form of sedation. The propofol FDA labeling and potential medicolegal consequences of sedation-related complications were a major impediment to widespread adoption of this technique by endoscopists.

At the same time, efforts were made to use a newer anesthetic, fospropofol, thereby overcoming the regulatory hurdle of propofol. Fospropofol is a prodrug of propofol with a slightly slower pharmacokinetic clearance [31], to be expected as the drug undergoes hydrolysis by alkaline phosphatase before releasing propofol, the active form. Although sedation produced with fospropofol was dosedependent and acceptable in the performance of GI endoscopy, its cardiac and respiratory side effects were largely similar. Further, even though the drug was devoid of any local pain on injection, it caused unacceptable perineal pain and pruritus [32]. The last hurdle was the same as with propofol. The FDA labeling for fospropofol (Lusedra) stated that it "should be administered only by persons trained in the administration of general anesthesia and not involved in the conduct of the diagnostic or therapeutic procedure" [33]. Although the drug was first approved by the FDA on December 12, 2008, it was soon discontinued [34].

The final attempt to resurrect non-anesthesia provideradministered propofol came in the form of SEDASYS $\left({ }^{\circledR}\right)$. 
This is a computer-assisted personalized sedation system that enables non-anesthesia providers to administer minimal-to-moderate propofol-fentanyl only sedation, simultaneously integrating comprehensive patient monitoring and drug delivery, personalized to the needs of the individual patient [35]. There was only one randomized comparative study on the safety and utility of SEDASYS before FDA approval [36]. In this non-blinded multicenter study, Pambianco et al. randomized four hundred ninetysix patients to SEDASYS System (SED) and 504 to benzodiazepine/opioid combination. Although they concluded that the SEDASYS system, when used by endoscopist/ nurse teams, could provide a safe and effective on-label means to administer propofol, the comparison group was not appropriate. Rather than a comparison with sedation by anesthesia providers, they compared the system to conscious sedation. Clearly, both patients and endoscopists were more comfortable with the deeper degrees of sedation that that was provided by SEDASYS. Although the device was approved to provide only minimal-moderate sedation during routine colonoscopy and EGD, inadequate sedation and inability to complete the procedure was always a concern. In an effort to obtain FDA approval, the manufacturers were forced to compromise on the depth of sedation. The manufacturers (Ethicon Inc) announced on March 10, 2016, that it was pulling SEDASYS out of the market. The reluctance of the FDA to approve anything that provides more than mild-moderate sedation and patients continuing to expect deep sedation was mutually incompatible [35, 37-40].

\section{Economic}

As discussed above, the cost of health care in any country must be carefully scrutinized in order to sustain any delivery model. The USA already spends $>17.8 \% \%$ of GDP toward health care, significantly higher than in many other developed countries such as the UK, Sweden, Germany, France, or Italy [41, 42] despite that the increased longevity predictions at birth (one of the indicators of overall national health) is higher in many of these countries than in the USA.

The cost of sedation is related to both drugs and the providers administering these drugs and monitoring the patients. A unique feature of the American health care is the requirement that anesthesia providers administer propofol, the drug considered as sine qua non for MAC. In the absence of any concrete data to support the increased safety of such practice, it is legitimate to debate this requirement. In fact, our own metanalysis suggested that anesthesia provider-administered propofol sedation is less safe than when administered by non-anesthesia providers such as nurses and gastroenterologists. Patient and gastroenterologist satisfaction was slightly lower when sedation was administered by non-anesthesia providers, likely related to the higher doses used by anesthesia accounting for increased frequency of adverse events and greater satisfaction. Nevertheless, one has to debate the financial burden of such a practice.

The economics of the US health-care system creates unique challenges. In a health-care model where insurance companies pay for the services, patients' expectations could be different. Additionally, with the high prevalence of litigation in the USA, gastroenterologists understandably are reluctant to take risks. Moreover, in the USA, the physician payments depend on the volume and complexity of the care delivered whereas in most other countries physicians are usually salaried. Even in academic institutions, there is an incentive based on how much a hospital can bill for their services.

The situation is further complicated by a few other factors. Most hospitals and endoscopy centers perform a combination of advanced and routine procedures. Most advanced procedures are performed under deep sedation provided by anesthesia providers. Moreover, many patients who need routine screening procedures are unsuitable for conscious sedation. Besides, conscious sedation is occasionally insufficient or becomes unsafe while the procedure is in progress. As a result, the presence of anesthesia providers becomes mandatory. Since in many hospitals the anesthesia providers are salaried, it would be less cost-effective if their services were utilized selectively. Thus, the reluctance of anesthesia providers to intervene in the midst of a failed conscious sedation is understandable. Lastly, endoscopists do not benefit from additional remuneration irrespective of administering either conscious sedation or propofol sedation. Endoscopists trained in the last decade have very little experience or exposure to conscious sedation. Yet, they cannot escape from any medicolegal liability associated with sedation-related adverse events. As a result of all of these factors, it is more cost-effective to utilize the services of anesthesia providers for all cases instead of for select patients.

There is no easy answer that addresses the above medical, political, and economic factors. The longer the status quo continues, the harder it will be to change or challenge. Any major change in insurance reimbursements might herald new approaches. Recently, the COVID-19 pandemic has challenged many of these practices with changes that are unlikely to last beyond the pandemic, as discussed in [43-46].

\section{Future of GI Endoscopic Sedation}

Any significant change in approach to GI endoscopic sedation is likely to come through new inventions. Fortunately, research in this part has been relentless. Any alternative to propofol sedation that is equally effective as propofol and 
safer in the hands of non-anesthesia providers will shift the paradigm. The following two drugs, especially when used in appropriate dosing, are likely to challenge the status quo in a significant way:

\section{Oliceridine}

Oliceridine belongs to a new class of opioids that are $\mathrm{G}$ protein-based $\mu$ receptor agonists [47]. Drugs such as fentanyl bind to the $\mu$-opioid receptor (a G protein-coupled receptor), activating two downstream pathways: the $\mathrm{G}$ protein-mediated pathway, responsible for analgesia, and the $\beta$-arrestin pathway, responsible for many adverse effects such as respiratory depression and GI dysfunction [48]. Oliceridine is proposed to achieve adequate analgesia with limited opioidrelated adverse events such as nausea, vomiting, sedation, constipation, reward/euphoria, dependence/withdrawal and respiratory depression. After binding to the $\mu$-opioid receptor, oliceridine selectively activates the $G$ protein-coupled signaling pathway while avoiding the $\beta$-arrestin pathway. Since one of the major limitations of traditional opioids such as fentanyl is respiratory depression, the use of oliceridine will likely enable the use of higher doses of midazolam without fear of respiratory depression. It is also likely to be beneficial in procedures such as ERCP since it has minimum GI side effects. Currently, the drug awaits FDA approval [49].

\section{Remimazolam}

One of the main advantages of propofol is quicker and clear-headed recovery followed by early discharge from the post-procedure recovery area, principally the result of its pharmacokinetics. The drug is metabolized quickly when administered in the doses and durations typically used for endoscopic sedation. Remimazolam is a benzodiazepine and like propofol acts on GABA-alpha receptors [50, 51]. In fact, it has a structure quite similar to midazolam, the very popular sedative in GI endoscopy. The main difference is the metabolism: while midazolam is chiefly metabolized in the liver and accumulates over time, wake up can be slow. Since remimazolam is metabolized independently of specific organs, it behaves more like propofol than midazolam. In the most recent randomized double-blind comparison of remimazolam to placebo for outpatient colonoscopy, Rex et al. demonstrated faster recovery of neuropsychiatric function compared with placebo (with midazolam rescue) and midazolam alone with a low but significant failure rate of $2 \%$ [52]. Remimazolam is still many months away from possible FDA approval [53].

It is quite likely that the remimazolam-oliceridine combination will reduce the use of propofol, consequently substantially decreasing the need for anesthesia providers in GI endoscopy.

\section{Conclusions}

The use of sedation and the involvement of anesthesia providers in GI endoscopy have evolved extensively over the last 3 decades. Since the issues surrounding endoscopic sedation are extremely complicated, they cannot be resolved with a single solution. Yet, the paradigm where all patients are administered propofol by anesthesia providers is clearly unsustainable. The way forward is perhaps a hybrid model that includes conscious sedation, propofol sedation, and general anesthesia used appropriately. Future drug innovations, especially the availability of oliceridine and remimazolam, might also be the breakthrough that will positively change the field forever.

\section{Compliance with Ethical Standards}

Conflict of interest The authors declare that they have no conflicts of interest.

\section{References}

1. Lin OS. Sedation for routine gastrointestinal endoscopic procedures: a review on efficacy, safety, efficiency, cost and satisfaction. Intest Res. 2017;15:456-466.

2. Ladas SD, Aabakken L, Rey J-F, et al. Use of sedation for routine diagnostic upper gastrointestinal endoscopy: a European Society of Gastrointestinal Endoscopy Survey of National Endoscopy Society Members. Digestion. 2006;74:69-77.

3. Garg PK, Singh AP, Jain BK, Bansal A, Mohanty D, Agrawal V. Safety and acceptance of non-sedated upper gastrointestinal endoscopy: a prospective observational study. J Laparoendosc Adv Surg Tech. 2012;22:315-318.

4. Suzuki T, Kitagawa Y, Nankinzan R, Yamaguchi T. Early gastric cancer diagnostic ability of ultrathin endoscope loaded with laser light source. World J Gastroenterol. 2019;25:1378-1386.

5. Iqbal N, Ramcharan S, Doughan S, Shaikh I. Colonoscopy without sedation: patient factors alone are less likely to influence its uptake. Endosc Int Open. 2016;4:E534-E537.

6. Terruzzi V, Paggi S, Amato A, Radaelli F. Unsedated colonoscopy: a neverending story. World J Gastrointest Endosc. 2012;4:137-141.

7. Ticktin HE, Trujillo NP. Evaluation of diazepam for pre-endoscopy medication. Am J Dig Dis. 1965;10:979-984.

8. Bianchi Porro G, Baroni S, Parente F, Lazzaroni M. Midazolam versus diazepam as premedication for upper gastrointestinal endoscopy: a randomized, double-blind, crossover study. Gastrointest Endosc. 1988;34:252-254.

9. Ferreira AO, Cravo M. Sedation in gastrointestinal endoscopy: where are we at in 2014? World J Gastrointest Endosc. 2015;7:102-109.

10. Etzkorn KP, Diab F, Brown RD, et al. Endoscopic retrograde cholangiopancreatography under general anesthesia: indications and results. Gastrointest Endosc. 1998;47:363-367.

11. An Astounding 19 Million Colonoscopies are Performed Annually in The United States [Internet]. iData Research. 2018 [cited 2020 May 23]. Available from: https://idataresearch.com/ 
an-astounding-19-million-colonoscopies-are-performed-annua lly-in-the-united-states/.

12. Can We Redefine the Term 'Monitored Anesthesia Care'? [Internet]. Anesthesia Experts. 2017 [cited 2020 May 19]. Available from: https://anesthesiaexperts.com/uncategorized/ redefine-term-monitored-anesthesia-care/.

13. Mandel JE, Tanner JW, Lichtenstein GR, et al. A randomized, controlled, double-blind trial of patient-controlled sedation with propofol/remifentanil versus midazolam/fentanyl for colonoscopy. Anesth Analg. 2008;106:434-439. (table of contents).

14. Ng JM, Kong CF, Nyam D. Patient-controlled sedation with propofol for colonoscopy. Gastrointest Endosc. 2001;54:8-13.

15. Ulmer BJ, Hansen JJ, Overley CA, et al. Propofol versus midazolam/fentanyl for outpatient colonoscopy: administration by nurses supervised by endoscopists. Clin Gastroenterol Hepatol Off Clin Pract J Am Gastroenterol Assoc. 2003;1:425-432.

16. Sipe BW, Rex DK, Latinovich D, et al. Propofol versus midazolam/meperidine for outpatient colonoscopy: administration by nurses supervised by endoscopists. Gastrointest Endosc. 2002;55:815-825.

17. Byrne MF, Chiba N, Singh H, Sadowski DC. Propofol use for sedation during endoscopy in adults: a Canadian Association of Gastroenterology position statement. Can J Gastroenterol. 2008;22:457-459.

18. Nishizawa T, Suzuki H. Propofol for gastrointestinal endoscopy. United Eur Gastroenterol J. 2018;6:801-805.

19. Shafer A, Doze VA, Shafer SL, White PF. Pharmacokinetics and pharmacodynamics of propofol infusions during general anesthesia. Anesthesiology. 1988;69:348-356.

20. Tabiri S, Adjimani NT, Mohammed BS. Propofol versus pethidine/midazolam sedation: benefits in flexible upper gastrointestinal endoscopy. Ain-Shams J Anesthesiol. 2018;10:11.

21. Koshy G, Nair S, Norkus EP, Hertan HI, Pitchumoni CS. Propofol versus midazolam and meperidine for conscious sedation in GI endoscopy. Am J Gastroenterol. 2000;95:1476-1479.

22. Goudra B, Nuzat A, Singh PM, Gouda GB, Carlin A, Manjunath AK. Cardiac arrests in patients undergoing gastrointestinal endoscopy: a retrospective analysis of 73,029 procedures. Saudi J Gastroenterol Off J Saudi Gastroenterol Assoc. 2015;21:400-411.

23. Goudra B, Nuzat A, Singh PM, Borle A, Carlin A, Gouda G. Association between type of sedation and the adverse events associated with gastrointestinal endoscopy: an analysis of 5 years' data from a Tertiary Center in the USA. Clin Endosc. 2016;50:161.

24. Wadhwa V, Issa D, Garg S, Lopez R, Sanaka MR, Vargo JJ. Similar risk of cardiopulmonary adverse events between propofol and traditional anesthesia for gastrointestinal endoscopy: a systematic review and meta-analysis. Clin Gastroenterol Hepatol. 2017;15:194-206.

25. Vargo JJ, Niklewski PJ, Williams JL, Martin JF, Faigel DO. Patient safety during sedation by anesthesia professionals during routine upper endoscopy and colonoscopy: an analysis of 138 million procedures. Gastrointest Endosc. 2017;85:101-108.

26. Rex DK, Heuss LT, Walker JA, Qi R. Trained registered nurses/ endoscopy teams can administer propofol safely for endoscopy. Gastroenterology. 2005;129:1384-1391.

27. Clarke AC, Chiragakis L, Hillman LC, Kaye GL. Sedation for endoscopy: the safe use of propofol by general practitioner sedationists. Med J Aust. 2002;176:158-161.

28. Heuss LT, Schnieper P, Drewe J, Pflimlin E, Beglinger C. Safety of propofol for conscious sedation during endoscopic procedures in high-risk patients - a prospective, controlled study. Am J Gastroenterol. 2003;98:1751-1757.

29. Cohen LB, DeLegge MH, Aisenberg J, et al. AGA Institute review of endoscopic sedation. Gastroenterology. 2007;133:675-701.
30. Vargo JJ, Cohen LB, Rex DK, Kwo PY. Position statement: nonanesthesiologist administration of propofol for GI endoscopy. Gastrointest Endosc. 2009;70:1053-1059.

31. Levitzky BE, Vargo JJ. Fospropofol disodium injection for the sedation of patients undergoing colonoscopy. Ther Clin Risk Manag. 2008;4:733-738.

32. Pergolizzi JV, Gan TJ, Plavin S, Labhsetwar S, Taylor R. Perspectives on the role of fospropofol in the monitored anesthesia care setting. Anesthesiol Res Pract. 2011;2011:e458920.

33. Drug Approval Package: Lusedra (Fospropofol Disodium) NDA \#022244 [Internet]. [cited 2020 May 21]. Available from: https:// www.accessdata.fda.gov/drugsatfda_docs/nda/2008/022244s000 TOC.cfm.

34. Lusedra (fospropofol disodium) FDA Approval History [Internet]. Drugs.com. [cited 2020 May 21]. Available from: https://www. drugs.com/history/lusedra.html.

35. Goudra BG, Singh PM, Chandrasekhara V. SEDASYS(®), airway, oxygenation, and ventilation: anticipating and managing the challenges. Dig Dis Sci. 2014. https://doi.org/10.1007/s1062 0-013-2996-z.

36. Pambianco DJ, Vargo JJ, Pruitt RE, Hardi R, Martin JF. Computer-assisted personalized sedation for upper endoscopy and colonoscopy: a comparative, multicenter randomized study. Gastrointest Endosc. 2011;73:765-772.

37. Goudra BG, Singh PM. SEDASYS, sedation, and the unknown. $J$ Clin Anesth. 2014;26:334.

38. Goudra B, Singh PM. Failure of Sedasys: destiny or poor design? Anesth Analg. 2016;124:686-688.

39. Martin JF, Bridenbaugh P, Gustafson M. The SEDASYS System is not intended for the sedation of high-risk patients. Gastrointest Endosc. 2011;74:723.

40. Ethicon Pulling Sedasys Anesthesia System [Internet]. [cited 2016 Mar 10]. Available from: http://www.outpatientsurgery.net/ news/2016/03/10/ethicon-pulling-sedasys-anesthesia-system.

41. U.S. health expenditure as GDP share 1960-2019 [Internet]. Statista. [cited 2020 May 23]. Available from: https://www.stati sta.com/statistics/184968/us-health-expenditure-as-percent-ofgdp-since-1960/.

42. Healthcare expenditure statistics - Statistics Explained [Internet]. [cited 2020 May 23]. Available from: https://ec.europa.eu/euros tat/statistics-explained/index.php/Healthcare_expenditure_stati stics.

43. Elli L, Rimondi A, Scaramella L, et al. Endoscopy during the Covid-19 outbreak: experience and recommendations from a single center in a high-incidence scenario. Dig Liver Dis. 2020;52:606-612.

44. Gralnek IM, Hassan C, Dinis-Ribeiro M. COVID-19 and endoscopy: implications for healthcare and digestive cancer screening. Nat Rev Gastroenterol Hepatol. 2020;13:1-3.

45. Furfaro F, Vuitton L, Fiorino G, et al. SFED recommendations for IBD endoscopy during COVID-19 pandemic: Italian and French experience. Nat Rev Gastroenterol Hepatol. 2020;11:1-10.

46. Hayee B, Thoufeeq M, Rees CJ, Penman I, East J. Safely restarting GI endoscopy in the era of COVID-19. Gut [Internet]. 2020 Jun 5 [cited 2020 Jun 27]; Available from: https://gut.bmj.com/conte nt/early/2020/06/05/gutjnl-2020-321688.

47. Kobilka BK. G protein coupled receptor structure and activation. Biochim Biophys Acta. 2007;1768:794-807.

48. Raehal KM, Bohn LM. $\beta$-arrestins: regulatory role and therapeutic potential in opioid and cannabinoid receptor-mediated analgesia. Handb Exp Pharmacol. 2014;219:427-443.

49. Goudra B, Gouda G, Mohinder P. Recent developments in drugs for GI endoscopy sedation. Dig Dis Sci. 2020;. https://doi. org/10.1007/s10620-020-06044-5.

50. Saari TI, Uusi-Oukari M, Ahonen J, Olkkola KT. Enhancement of GABAergic activity: neuropharmacological effects of 
benzodiazepines and therapeutic use in anesthesiology. Pharmacol Rev. 2011;63:243-267.

51. Goudra B, Singh P. Remimazolam: the future of its sedative potential. Saudi J Anaesth. 2014;8:388.

52. Rex DK, Bhandari R, Desta T, et al. A phase III study evaluating the efficacy and safety of remimazolam (CNS 7056) compared with placebo and midazolam in patients undergoing colonoscopy. Gastrointest Endosc. 2018;88:427.e6-437.e6.

53. FDA accepts filing of NDA for Remimazolam [Internet]. [cited 2019 Sep 10]. Available from: https://www.cosmopharma.com/ news-and-media/press-releases-and-company-news/2019/19061 0.

Publisher's Note Springer Nature remains neutral with regard to jurisdictional claims in published maps and institutional affiliations. 